\section{Time of Day and Performance on Cognitive Tests in Patients with Mild Dementia}

\section{Upinder Singh ${ }^{1 *}$, Mandeep Gill², Rebecca Rice ${ }^{2}$, Fallon Dimaano $^{2}$, Andrea Warburton ${ }^{2}$ and Michael R Wells ${ }^{2 *}$}

${ }^{1}$ Department of Primary Care, Touro University Nevada, College of Osteopathic Medicine, American Pacific Drive, Henderson, Nevada, USA

${ }^{2}$ Department of Basic Sciences, Touro University Nevada, College of Osteopathic Medicine, American Pacific Drive, Henderson, Nevada, USA

\begin{abstract}
Background

Cognitive screening for elderly patients with mild dementia is typically conducted in the morning under the impression that testing at this time will optimize performance and cooperation of patients. This study was conducted to determine if the time of day in which several cognitive screening tests would be normally given could significantly affect test performance in patients with mild dementia and normal control subjects.

\section{Methods}

Fifty nursing home residents with mild to moderate dementia and twenty normal control subjects were given three commonly used cognitive tests in two separate sessions (morning and afternoon) with a two week interval between sessions. Half of subjects were tested first in the morning and second in the afternoon, the other half first in the afternoon and second in the morning. Evaluation tools included the Mini Mental Status Exam (MMSE), the Mini-Cog Test, and Semantic Verbal Fluency (for animal names) at each session. Test scores were compared within each subject between the morning and afternoon sessions.

Results

Across all subjects, better scores were observed for afternoon performance in the MMSE score $(p<0.005)$ and Mini-Cog $(p<0.011)$ for subjects who tested first in the morning and second in the afternoon. No differences were observed in the reverse condition. The morning/afternoon differences and trends across all subjects
\end{abstract}

*Corresponding authors: Michael R Wells, Department of Basic Sciences Touro University Nevada, College of Osteopathic Medicine, 874 American Pacific Drive, Henderson, Nevada 89014, USA, Tel: +1 7027771810; Fax: +1 7027771799; E-mail: Michael.Wells@tun.touro.edu

Upinder Singh, Department of Primary Care, Touro University Nevada, College of Osteopathic Medicine, American Pacific Drive, Henderson, Nevada, USA, Tel: +1 7023532024; E-mail: usinghmd@yahoo.com

Citation: Singh U, Gill M, Rice R, Dimaano F, Warburton A, et al. (2016) Time of Day and Performance on Cognitive Tests in Patients with Mild Dementia. $J$ Alzheimers Neurodegener Dis 2: 003.

Received: December 31, 2015; Accepted: February 23, 2016; Published: March 09, 2016 were strongest for the mild dementia group (MMSE, $p<0.003$; Mini-Cog, $p<0.075$ ). However, the analysis also indicated that test experience contributed to some of the observed differences, such that no clear effect of time of day on performance could be substantiated.

\section{Conclusion}

Under the conditions of this study, we could not conclude that performance on cognitive tests was significantly affected by the time of day of administration. A more comprehensive study will be necessary to better define the potential factors identified.

Keywords: Circadian; Cognition; Dementia; Performance

\section{Introduction}

Brief mental status examinations are often applied by physicians and other healthcare professionals in managed care settings as screening to identify and document basic mental status and relative level of cognitive impairment [1]. With the inclusion of cognitive assessment as a required part of the affordable care act annual wellness visit for medicare enrollees [2], attention to the process of such screening has increased [3]. Patient performance on such tests can have important implications for treatment in addition to raising potential legal issues. This is particularly true for patients with mild dementia, where the ability to make medical or life decisions may come into question. For such individuals, any significant factor in judging cognitive capacity could affect decisions.

It is known that cognitive performance on particular tasks can vary with circadian cycles, especially when comparing performance during waking with time during which sleep would normally occur [4]. Beyond the dramatic differences in cognitive performance demonstrable at the extremes of the circadian cycle, the extent to which the time of day could affect performance on some standard cognitive tests normally used to assess cognitive status in mildly demented patients has not been studied. It is generally presumed that midmorning testing is optimal, since normal adults have optimal performance on most cognitive tasks at this time [4]. However, long term care residents can suffer from sleep disturbances attributed to a number of factors including medical and psychiatric disorders, medications, circadian rhythm disturbances, sleep disordered breathing, environmental conditions and lifestyle habits [5]. These sleep disturbance scan alter patterns of cognitive performance [6] and general patterns of activity [7]. As factors such as sleep disturbance can significantly affect cognitive performance, optimal times of day in which to test these individuals could differ from the normal expectation [6]. Other related phenomena, such as "sundowning" [8], also contribute to a perception that midmorning is an optimal time to administer cognitive testing in these individuals.

We wished to address the potential impact of time of day in which cognitive testing is administered to older adults with mild dementia on a practical scale (i.e., during a normal workday cycle; morning to afternoon) as might be encountered in a long term or assisted living facility environment. Mental status was assessed using some commonly used brief cognitive tests (Mini-Mental State Exam (MMSE), Mini-Cog, and Semantic Verbal Fluency (SVF). Fifty 
elderly adults with mild dementia and 20 normal control subjects were used to evaluate mental status at two times during a normal workday, morning and afternoon, in a crossed design. The results suggest that there is no clear advantage to morning testing for cognitive performance as measured by these commonly administered tests. Test experience is also a significant factor which must be controlled [4].

\section{Methods}

\section{Subjects}

Subjects were recruited under an Institutional Review Board approved protocol from five assisted living facilities in the Las Vegas, Nevada area and local university staff. All subjects (or their legal representatives) signed an informed consent. The extent to which subjects were allowed to consent for the study themselves was determined by the policies of facility in which they resided. Fifty female and male subjects ( 31 females, 19 males) were recruited as the mild dementia group. These criteria required subjects to be over 60 years of age (Average age $85 \pm 7.1$ years) with a diagnosis of mild to moderate dementia (Mini-Mental State Exam score between 11 and 27). Subjects rated outside of this range were excluded. Control subjects were recruited from University staff and included 20 subjects (16 females, 4 males) between the ages of 27 and 66 (average age $47.7 \pm 12.5$ years). Control subjects were considered cognitively sound with MMSE scores between 28 and 30. Exclusion criteria for the control group included a diagnosis of dementia, schizophrenia, bipolar disease, or intellectual disability. Exclusion criteria for both groups included unwillingness to sign the consent form and/or uncooperativeness while performing tests.

\section{Evaluation methods}

Cognitive performance was assessed using three commonly used tests, the Mini-Mental State Examination (MMSE; 30 points possible [9]), the Mini-Cog (7 points possible [10]) and semantic verbal fluency using animal names (19-30 considered normal [11]). All tests were administered twice to all subjects, once in the morning (between 8:30AM and $11 \mathrm{AM}$ ) and once in the afternoon to evening (3PM to $6 \mathrm{PM}$ ) with a period of two weeks between the testing sessions. To control for test experience ("practice effect" [4]), both experimental and control groups were randomly divided into 2 subgroups: one tested first in the morning and then in the afternoon the other tested first in the afternoon and then in the morning.

Demographic information on age, gender, ethnicity, educational level (did not complete high school, did complete high school, attended college), memory medications (Namenda, Exelon, Aricept), medical conditions (diabetes, stroke, depression), and time in current facility (if subject was a resident) was gathered from each participant. This information was collected largely to identify potential factors in the non-random distribution of subjects as the data was analyzed.

\section{Data analysis}

Statistical analysis was performed using Sigma Plot Version 12.5 statistical software purchased from Systat Software Inc., San Jose, CA. A two-way analysis of variance with repeated measures was used as the primary test for significance of morning to afternoon differences for the individual tests (MMSE, Mini-Cog, Semantic Verbal Fluency). Variables included cognitive test, experimental and control groups, morning scores, afternoon scores, and time of first testing. All subjects (mild dementia and control) were examined first for possible overall differences. Since each subject acted as his/her own control for test time differences, the data, where appropriate, was expressed as score differences between the second and first (2nd-1st) tests. This expression of data was chosen since, under the conditions of test timing, second scores were usually higher (score differences positive). Statistical significance was considered to be less than 0.05 probability of equivalence in comparisons.

\section{Results}

Control and Mild Dementia Baseline Comparisons-The average MMSE Score differed significantly $(\mathrm{p}<0.001)$ between the control $(29.2 \pm 1.2)$, and experimental groups (19.4 \pm 0.7$)$. The MMSE scores did not differ between males and females in either group.

Over all subjects (mild dementia and control), there were no significant differences in the scores of subjects who took the tests in the Morning First (MF) compared to those who took their first test in the Afternoon (AF) in the scores for MMSE, Mini-Cog, or SVF ( $p>0.05$ in each case). This was important to establish the uniformity of groups (Figure 1).

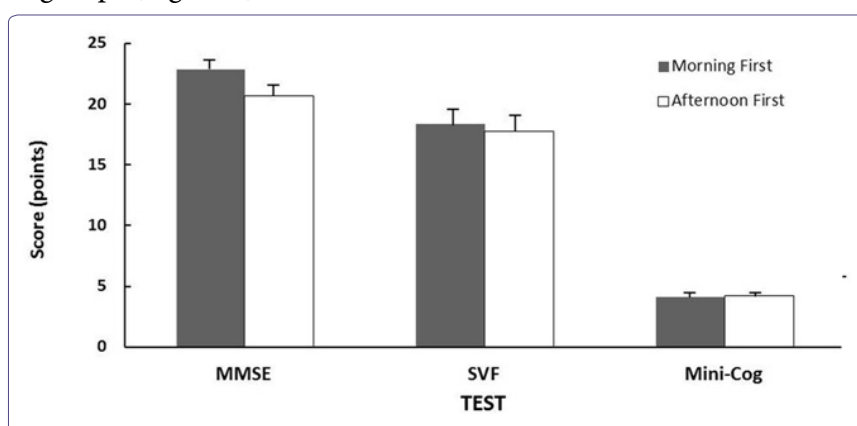

Figure 1: Test score averages ( \pm Standard Error) over all subjects for groups in which tests were given in the morning first or afternoon first. MMSE-MiniMental State Exam, SVF-Semantic Verbal Fluency Test. There were no significant differences for any test between the groups.

An examination of demographic factors (gender, education, age, use of medications) indicated a relatively even distribution of factors across groups. There were some expected correlations of demographic factors with performance scores on the tests given, such as an inverse correlation of MMSE scores with age and a positive correlation with education. However, there was no clear correlation of the demographic factors collected to the magnitude of differences between the first and second tests for individuals within groups, suggesting that within subject differences were not systematically affected.

We next examined the combined group (mild dementia and control) for a possible effect of when tests were taken first (morning or afternoon) on score differences between the two testing sessions (Figure 2). Subjects who received their first test in the morning had significantly higher scores on their second test given in the afternoon on two of the three tests applied. Significant differences were observed for the MMSE $(p<0.005)$ and the Mini-Cog $(p<0.011)$. These score differences were small (3-10\%), but consistent within subjects. Semantic verbal fluency testing showed a similar trend, but had a higher variability in scores $(\mathrm{p}=0.276$; Figure 2$)$. There were no significant differences in scores between the testing sessions for the combined group that received their first testing session in the afternoon, and second in the morning (reverse order). However, there was a trend in this combined group for higher average scores on the second test session compared to the first (Figure 2). MMSE scores gave the most pronounced trend, where tests given in the afternoon first were close to being significantly lower than the second test 
given in the morning $(\mathrm{p}<0.053)$. The indication of higher scores on the second of two tests for both groups suggested test experience could be contributing to score differences observed.

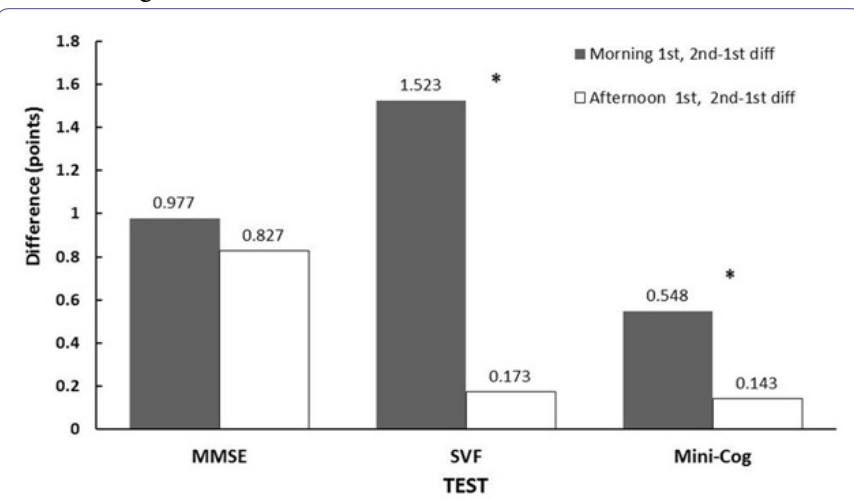

Figure 2: Average Test Score Differences for all subjects (mild dementia and control) between the first and second administration times (2nd-1st). Asterisks $\left({ }^{*}\right)$ show differences which were significant within subjects $(p<0.05)$ analyzed by the paired testing procedure described. MMSE-Mini-Mental State Exam, SVF-Semantic Verbal Fluency Test.

\section{Mild dementia and control groups}

Comparisons of data from the mild dementia and control groups separately indicated that the significant within subject differences observed in test scores for the groups together were largely derived from the mild dementia group data. In the control group alone, there were no differences between the scores for first and second administration of tests regardless of the order for any of the tests applied (Figure 3).

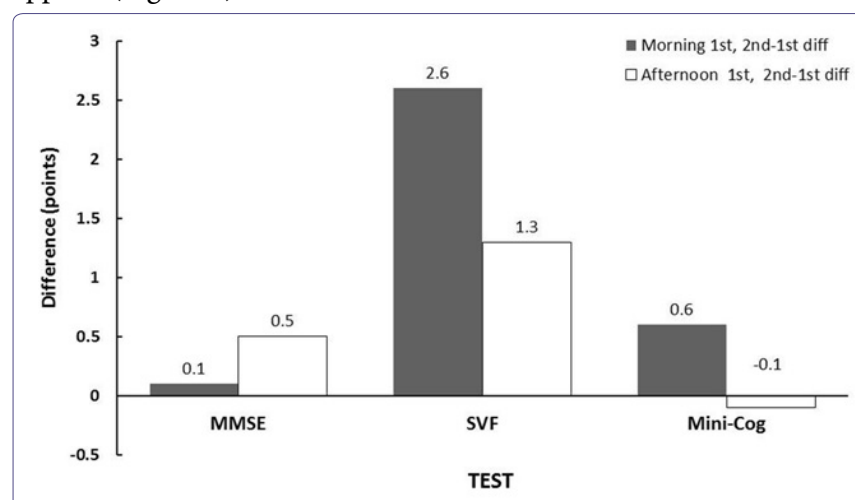

Figure 3: Average Test Score differences for control subjects between the first and second administration times (2nd-1st) analyzed by the paired testing procedure described. There were no differences within subjects for any of the tests. MMSE-Mini-Mental State Exam, SVF-Semantic Verbal Fluency Test.

In the subjects with mild dementia, there was a significant difference between the scores of MMSE tests given in the morning first and the second test in the afternoon $(\mathrm{p}<0.005)$. A similar trend in the Mini-Cog scores were observed ( $p=0.086$; Figure 4$)$. There were no significant differences in scores for tests given in the afternoon first, but again, a trend $(\mathrm{p}<0.1)$ for second administration scores to be higher with the MMSE.

The significant advantage of afternoon cognitive performance in patients with mild dementia appeared to be at least partially offset by test experience. To examine the net effect, the magnitude of the difference between first versus second test scores in groups tested in the morning first or afternoon first was compared (ANOVA). There were no differences in the magnitude of score change for any morning first or afternoon first group pair (mild dementia, control,

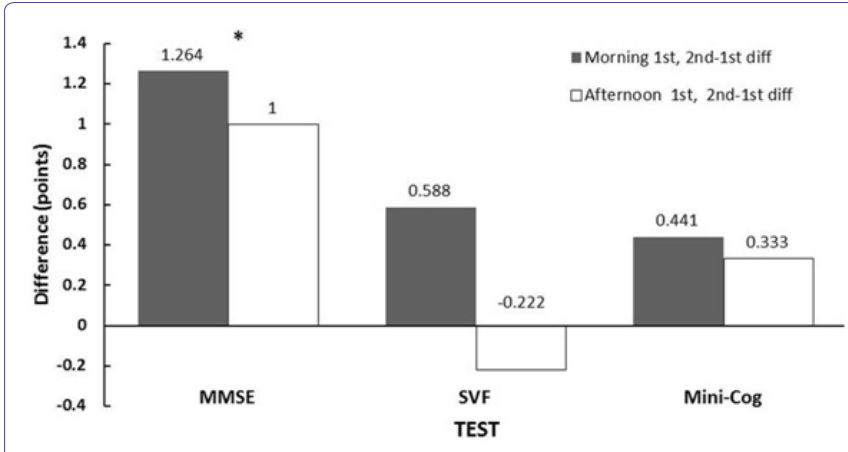

Figure 4: Test score differences for subjects with mild dementia in which tests were given in the morning first or afternoon first. Asterisks $\left(^{*}\right)$ show differences that were significant within subjects $(p<0.05)$ analyzed by the paired testing procedure described. MMSE-Mini-Mental State Exam, SVF-Semantic Verbal Fluency Test.

both groups together). This indicated the time of initial testing (morning or afternoon) had no significant influence on test performance, once the confounding factor of test experience was removed.

\section{Discussion}

We interpret these data to indicate that the time of test administration during the day and test experience may both have small, but potentially measurable influences on the observed test scores for two commonly used cognitive screening tests for dementia (MMSE and Mini-Cog). These influences were suggested, but could not be fully characterized in the present study, due to the limited sample size and the potentially offsetting effects of test experience and a potential advantage of afternoon administration. Larger sample sizes and greater statistical power will be necessary to better define the influences for the relatively small effects observed.

The evidence for an effect of administration time during the day on these cognitive screening tests was indicated by small, but significant increases in the scores of all subjects (mild dementia and control) who were tested first in the morning followed in two weeks by the same tests administered in the afternoon. Test scores differed significantly for the MMSE and Mini-Cog, but not semantic verbal fluency. The MMSE appeared to be the most sensitive to show these internal score differences.

No significant differences were seen in a separate group of subjects tested in the reverse order. The control group was included in the initial analysis as a mechanism to identify data trends in all subjects. The analysis indicated that the inclusion of the control group strengthened the statistical significance of the higher scores seen in tests given the afternoon, particularly for the Mini-Cog. This suggests that both groups may actually exhibit similar trends in test score differences, but that particularly the control group was not large enough to show the effect independently. The nature of scoring in the cognitive screening tests used also diminishes the range of score differences for control subjects, since most subjects in the normal range may have perfect or near perfect scores. The only open ended, test, semantic verbal fluency, had too a high variability within subjects to show a significant effect.

In the analysis of the separate groups of subjects with mild dementia and normal control groups, the normal control group alone did not show any differences between the two test sessions administered in either order. However, the subjects with mild dementia demonstrated a significant score difference between the 
morning and afternoon MMSE for subjects given the test first in the morning, but not for subjects given tests in the afternoon first. The size of this effect was about $5 \%$ of the score, but consistent. This finding is contrary to a general presumption that cognitive screen testing in the afternoon may present a disadvantage for patients with mild dementia as they tire from daily activities or begin to exhibit agitated behavior [12].

Test experience was suggested as a diluting factor for first to second test score differences, although we did not see a significant effect. Over all subjects (mild dementia and control), average test scores were generally higher for the second test, regardless of whether the second test session was given in the morning or afternoon. Test or practice experience has been cited as a factor to consider when testing is repeated over time [4]. It was anticipated that test experience could be a factor in this study, but a second consideration was that changes in the health condition of the group with mild dementia might also occur. It was believed that assessing cognitive performance over a longer interval might begin to reflect a deterioration of condition in the mild dementia group. A two week time interval between the first and second test sessions was chosen as a compromise between these two possibilities.

The trend for higher scores on the second of the two test sessions at either time of day suggested that the significant increase in the afternoon scores for a second test in our subjects with mild dementia was partially attributable to test experience. We looked for a possible net effect of time of day without test experience by comparing the magnitude of test differences in morning first versus afternoon first groups. No significant differences were detected. It was concluded that while there may be a small advantage of afternoon testing, it could not be conclusively demonstrated within the limits of our study due to the potential offset of test experience.

In the current study, our object was to examine potential effects on performance of time of day administration for commonly used cognitive tests under the general conditions which might be practically experienced in the environment of a nursing home or assisted living facility. As we could not detect any large effect, it does not appear, from a practical point of view, that the time of day of test administration has any significant impact on test performance in the use of these screening tools. It is noteworthy, however, that there was no indication of an advantage for morning testing. These findings further suggest that, as cognitive screening becomes increasingly utilized for patient Annual Wellness visits [2], the time of day these test are administered within a normal work day should not be of major concern, but should be conducted as consistently as possible.

\section{Conclusion}

Within the confines of a normal work day, there is no clear effect of time of day of test administration for the scores of three commonly used cognitive screening tests for patients with mild dementia. Test experience should be considered a factor for score evaluation even when the administration of these tests is separated by a period of two weeks. A study with a larger group size will be required to achieve the statistical power necessary to resolve these effects, due to their small size and test performance variability.

\section{Acknowledgement}

The study was completed in partial fulfillment of the requirements for the Master of Health Science Degree for three of the authors. The project was approved by the Institutional Review Board, with written consent obtained as described from all participants. No conflicts of interest have been reported by any of the authors.

\section{References}

1. Borson S, Scanlan JM, Sadak T, Lessig M, Vitaliano P (2014) Dementia Services Mini-Screen: a simple method to identify patients and caregivers in need of enhanced dementia care services. Am J Geriatr Psychiatry 22: 746-755.

2. Cordell CB, Borson S, Boustani M, Chodosh J, Reuben D, et al. (2013) Alzheimer's Association recommendations for operationalizing the detection of cognitive impairment during the Medicare Annual Wellness Visit in a primary care setting. Alzheimers Dement 9: 141-150.

3. Borson S, Frank L, Bayley PJ, Boustani M, Dean M, et al. (2013) Improving dementia care: the role of screening and detection of cognitive impairment. Alzheimers Dement 9: 151-159.

4. Blatter K, Cajochen C (2007) Circadian rhythms in cognitive performance: methodological constraints, protocols, theoretical underpinnings. Physiol Behav 90: 196-208.

5. Martin JL, Ancoli-Israel S (2008) Sleep disturbances in long-term care. Clin Geriatr Med 24: 39-50.

6. Ancoli-Israel S (2009) Sleep and its disorders in aging populations. Sleep Med 1: 7-11.

7. Carvalho-Bos SS, Riemersma-van der Lek RF, Waterhouse J, Reilly T, van Someren EJ (2007) Strong association of the rest-activity rhythm with well-being in demented elderly women. Am J Geriatr Psychiatry 15: 92-100.

8. Roth HL (2012) Dementia and sleep. Neurol Clin 30: 1213-1248.

9. Tombaugh TN, Mclntyre NJ (1992) The mini-mental state examination: a comprehensive review. J Am Geriatr Soc 40: 922-935.

10. Borson S, Scanlan J, Brush M, Vitaliano P, Dokmak A (2000) The mini-cog: a cognitive 'vital signs' measure for dementia screening in multi-lingual elderly. Int J Geriatr Psychiatry 15: 1021-1027.

11. Tombaugh TN, Kozak J, Rees $L$ (1999) Normative data stratified by age and education for two measures of verbal fluency: FAS and animal naming. Arch Clin Neuropsychol 14: 167-177.

12. Volicer L, Harper DG, Manning BC, Goldstein R, Satlin A (2001) Sundowning and circadian rhythms in Alzheimer's disease. Am J Psychiatry 158: 704-711. 